American Journal of Applied Sciences 3 (4): 1795-1802, 2006

ISSN 1546-9239

(c) 2006 Science Publications

\title{
Intelligent Control for Self-erecting Inverted Pendulum Via Adaptive Neuro-fuzzy Inference System
}

\author{
${ }^{1}$ A.A. Saifizul, ${ }^{1}$ Z. Zainon, ${ }^{2}$ N.A Abu Osman, ${ }^{3}$ C.A. Azlan and ${ }^{4}$ U.F.S Ungku Ibrahim \\ ${ }^{1}$ Department of Mechanical Engineering, Faculty of Engineering \\ University of Malaya, 50603, Kuala Lumpur, Malaysia \\ ${ }^{2}$ Department of BioMedical Engineering, Faculty of Engineering \\ University of Malaya, 50603, Kuala Lumpur, Malaysia \\ ${ }^{3}$ Department of BioMedical-Imaging, Faculty of Medicine \\ University of Malaya, 50603, Kuala Lumpur, Malaysia \\ ${ }^{4}$ Center for Foundation Studies in Science, University of Malaya \\ 50603 Kuala Lumpur, Malaysia
}

\begin{abstract}
A self-erecting single inverted pendulum (SESIP) is one of typical nonlinear systems. The control scheme running the SESIP consists of two main control loops. Namely, these control loops are swing-up controller and stabilization controller. A swing-up controller of an inverted pendulum system must actuate the pendulum from the stable position. While a stabilization controller must stand the pendulum in the unstable position. To deal with this system, a lot of control techniques have been used on the basis of linearized or nonlinear model. In real-time implementation, a real inverted pendulum system has state constraints and limited amplitude of input. These problems make it difficult to design a swing-up and a stabilization controller. In this paper, first, the mathematical models of cart and single inverted pendulum system are presented. Then, the Position-Velocity controller is designed to swingup the pendulum considering physical behavior. For stabilizing the inverted pendulum, a TakagiSugeno fuzzy controller with Adaptive Neuro-Fuzzy Inference System (ANFIS) architecture is used to guarantee stability at unstable equilibrium position. Experimental results are given to show the effectiveness of these controllers.
\end{abstract}

Key words: Takagi-sugeno fuzzy, ANFIS, self-erecting inverted pendulum

\section{INTRODUCTION}

The self-erecting single inverted pendulum (SESIP) system is a challenging problem in the area of control systems. It is very useful to demonstrate concepts in linear control such as the stabilization of unstable systems. Besides, as a typical unstable nonlinear system, inverted pendulum system is often used as a benchmark for verifying the performance and effectiveness of a new control method because of the simplicities of the structure.

The control strategy of SESIP system is composed of the swing-up control of the pendulum and the stabilizing control of the whole system that consists of angular control of the pendulum at upright position and position control of the cart at origin of rail. First, swingup control is to bring the pendulum from the downwards position to the upright position. This is achieved when the motor is given voltage in the appropriate direction and magnitude to drive the cart back and forth along the extremely limited track length repeatedly until the pendulum is close to the upright position. Thereafter, stabilizing control is to balance the pendulum in the upright position. When the pendulum leans in one direction, the control algorithm will try to move the cart under it with appropriate speed and direction. In this case, the algorithm will take the inputs i.e. the pendulum angle and cart position measured by encoders, then tell the cart which way and how fast to move.

Until now, a lot of intelligent approaches about the swing-up and stabilizing control of inverted pendulum system have been proposed. Mikukcic and Chen ${ }^{[1]}$ extracted fuzzy rules for inverted pendulum control by fuzzy clustering method. Brock ${ }^{[2]}$ has presented the fuzzy PD controller in balancing the pendulum in upright position and then the tuning process is done using evolutionary algorithms. He has successfully proved the robustness of the controller in simulated experiment. Kandadai and Tien $^{[3]}$ presented neurofuzzy architecture to automatically generate a fuzzy knowledge based by a pseudo-supervised learning scheme. It takes more than $12 s$ and its structure is more complex. Kawaji and Maeda ${ }^{[4]}$ constructed a simple fuzzy controller that impeded the position control of the cart as a virtual target angle into the angular control of

Corresponding Author: Ahmad Saifizul Bin Abdullah, Department of Mechanical Engineering, Faculty of Engineering, University of Malaya, 50603 Kuala Lumpur, Malaysia, Tel: 60379674495, Fax: 60379675317, 
the pendulum, but the controller was difficult to completely stabilize a pendulum system within a short time.

Since most of the classical fuzzy approach will cause the exponential increase in complexity, Horikawa et $a l .{ }^{[5]}$, Lin and Lee ${ }^{[6]}$, Partricar and Provence ${ }^{[7]}$ and Takagi and Hayashi ${ }^{[8]}$ presented techniques incorporating neural network into Fuzzy system. Kyung and Lee ${ }^{[9]}$ presented a fuzzy controller, whose rule base was derived from three neural networks. Although the fuzzy controller can stabilize an inverted pendulum system in about $8.0 \mathrm{~s}$, it needs 396 rules. Sakai and Takahama $^{[10]}$ applied a nonlinear optimization method to train a fuzzy controller for stabilization. However, the controller spent more than $200.0 s$ on stabilizing an inverted pendulum system.

In this paper, a position-velocity (PV) is used for swing-up control while a data-driven Takagi-Sugeno Fuzzy model called ANFIS is employed for the stabilizing control. A common Takagi-Sugeno fuzzy model approach typically uses common sense knowledge of human experts which tends to be "trial and error" in tuning its membership functions and rules. It may be less suitable for control design. Therefore, the ANFIS controller which was developed by ${ }^{[11]}$ is used to solve the SESIP stabilizing problem. This method is proposed to construct the fuzzy model by combining information obtained from measured data i.e. training data and checking data with heuristic knowledge expressed in the form of rules. Initially, the expert knowledge is formulated as a collection of if-then rules and the associated membership functions. But then, these rules and membership functions can be fine-tuned by using numerical data. This model tends to be adaptive. It is able to adjust itself to accommodate new situation, especially changes in the dynamic behavior of the SESIP plant in order to achieve the desired performance objective.

Modeling of cart system: The cart system can be represented as follows:

$$
G(s)=\frac{x_{c}(s)}{V_{m}(s)}
$$

where $G(s)$ is open-loop transfer function, $x_{c}$ is cart position and $V_{m}$ is motor voltage. Applying Newton's second law of motion to the cart system and by assuming cart's Coulumb friction is neglected, the equation of motion can be represented as follows:

$M \ddot{x}_{c}(t)=F_{c}(t)-B_{e q} \dot{x}_{c}(t)-F_{a i}(t)$

where $F_{c}$ is cart driving force produced by the motor and $F_{a i}$ is inertial force due to the motor's armature in rotation and other parameters are given in Table 1. The driving froce, $F_{c}$, generated by the DC motor and Table 1: List of symbols and model parameters acting on the cart through the motor pinion can be expressed as:

$$
F_{c}(t)=\frac{\eta_{g} K_{g} T_{m}(t)}{r_{m p}}
$$

where $T_{m}$ is torque generated by the motor.

Using Kirchhoff's Voltage law, the following equation is obtained:

$V_{m}(t)-R_{m} I_{m}(t)-L_{m}\left(\frac{\partial}{\partial t} I_{m}(t)\right)-E_{\text {emf }}(t)=0$

However, since $L_{m} \ll R_{m}$, the motor inductance is disregard and yields:

$I_{m}(t)=\frac{V_{m}(t)-E_{\text {emf }}(t)}{R_{m}}$

Since the back-emf voltage created by the motor, $E_{\text {emf }}$, is proportional to the motor shaft velocity $w_{m}$, the equation is obtained as:

$I_{m}(t)=\frac{V_{m}(t)-K_{m} w_{m}(t)}{R_{m}}$

Moreover, in order to account for the DC motor electrical losses, the motor efficiency is introduced to calculate the torque generated by the DC motor:

$T_{m}(t)=\eta_{m} K_{t} I_{m}(t)$

Substituting (6) and (7) into (3) leads to:

$F_{c}(t)=\frac{\eta_{g} K_{g} \eta_{m} K_{t}\left(V_{m}(t)-K_{m} w_{m}(t)\right)}{R_{m} r_{m p}}$

By considering the rack and pinion and the gearbox mechanisms, the motor angular velocity can be written as a function of the cart linear velocity, as expressed by:

$w_{m}(t)=\frac{K_{g} \dot{x}(t)}{r_{m p}}$

Therefore, substituting (9) into (8) and rearranging leads to:

$F_{c}(t)=\frac{\eta_{g} K_{g} \eta_{m} K_{t}\left(r_{m p} V_{m}(t)-K_{g} K_{m} \dot{x}(t)\right)}{R_{m} r_{m p}{ }^{2}}$

As seen at the motor pinion, the armature inertial force due to the motor rotation and acting on the cart can be expressed as a function of the armature inertial torque:

$F_{a i}=\frac{\eta_{g} K_{g} T_{a i}}{r_{m p}}$

Applying Newton's second law of motion to the motor shaft:

$J_{m} \ddot{\theta}_{m}(t)=T_{a i}(t)$

where $\theta_{m}$ is motor shaft rotation angle. Moreover, the mechanical configuration of the cart's rack-pinion system gives the following relationship: 


\begin{tabular}{lll}
\hline Symbol & Description & Value/Unit \\
\hline$V_{m}$ & Motor nominal input voltage & $6 \mathrm{~V}$ \\
$R_{m}$ & Motor armature resistance & $2.6 \Omega$ \\
$L_{m}$ & Motor armature inductance & $0.18 \mathrm{mH}$ \\
$K_{t}$ & Motor torque constant & $0.00767 \mathrm{Nm} / \mathrm{A}$ \\
$\eta_{m}$ & Motor efficiency & $100 \%$ \\
$K_{m}$ & EMF constant & $0.00767 \mathrm{Vs} / \mathrm{rad}$ \\
$J_{m}$ & Rotor moment inertia & $3.9 \times 10^{-7} \mathrm{kgm}^{2}$ \\
$K_{g}$ & Planetary gearbox gear ratio & 3.71 \\
$\eta_{g}$ & Planetary gearbox efficiency & $100 \%$ \\
$r_{m p}$ & Motor pinion radius & $6.35 \times 10^{-3} \mathrm{~m}$ \\
$r_{p p}$ & Position pinion radius & $1.48 \times 10^{-2} \mathrm{~m}$ \\
$B_{e q}$ & Equivalent viscous damping coefficient at the motor pinion & $5.4 \mathrm{Nms} / \mathrm{rad}$ \\
$B_{p}$ & Viscous damping coefficient at the pendulum pivot & $0.0024 \mathrm{Nms} / \mathrm{rad}$ \\
$l_{p}$ & Pendulum length length from pivot to center of gravity & $0.3302 \mathrm{~m}$ \\
$I_{p}$ & Pendulum moment of inertia & $7.88 \times 10^{-3} \mathrm{~kg} \mathrm{~m} \mathrm{~m}^{2}$ \\
$M_{p}$ & Pendulum mass & $0.230 \mathrm{~kg}$ \\
$M$ & Cart mass & $0.94 \mathrm{~kg}$ \\
\hline
\end{tabular}

$$
\theta_{m}=\frac{K_{g} x}{r_{m p}}
$$

Substituting (11) and (12) into (10)

$$
F_{a i}=\frac{\eta_{g} K_{g}{ }^{2} J_{m} \ddot{x}(t)}{r_{m p}{ }^{2}}
$$

Finally, substituting (10) and (14) into (2), applying the Laplace transform and rearranging, yields the desired open loop transfer function for the cart system, such that:

$$
G(s)=\frac{r_{m p} \eta_{g} K_{g} \eta_{m} K_{t}}{\left(\left(R_{m} M r_{m p}{ }^{2}+R_{m} \eta_{g} K_{g}{ }^{2} J_{m}\right) s+\eta_{g} K_{g}{ }^{2} \eta_{m} K_{t} K_{m}+B_{e q} R_{m} r_{m p}{ }^{2}\right) s}
$$

Single inverted pendulum equation of motion: Angle definitions and schematic diagram of SESIP are shown in Fig. 1.

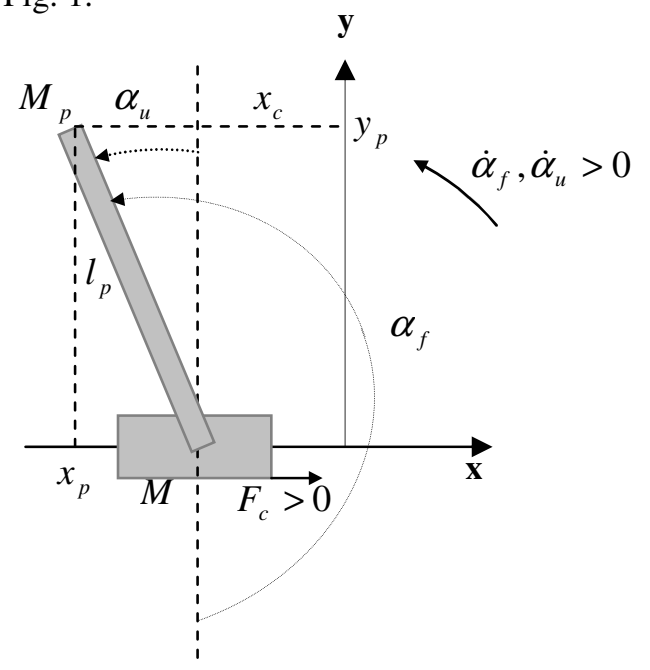

Fig. 1: SESIP schematic

The SIP system system is made of a cart on top of which pendulum is pivoted. The cart is constrained to move only in the horizontal $x$ direction, while the pendulum can only rotate in the $x-y$ plane. The SIP system has two degrees of freedom and can therefore be fully represented using two generalized coordinates: Horizontal displacement of the cart, $x_{c}$; and Rotational displacement of pendulum, $\alpha$. Assume the (nonlinear) Coulomb friction applied to the linear cart is assumed to be neglected. Moreover, the force on the linear cart due to the pendulum's action has also been neglected in the presently developed model. A nonlinear equation of motion (OEM) of single inverted pendulum (SIP) system can be derived using Lagrange's equation.

$\frac{d}{d t}\left[\frac{\partial L}{\partial \dot{x}_{c}}\right]-\frac{\partial L}{\partial x_{c}}=Q_{x_{c}}$

and

$\frac{d}{d t}\left[\frac{\partial L}{\partial \dot{\alpha}}\right]-\frac{\partial L}{\partial \alpha}=Q_{\theta}$

with $L=T_{T}-V_{T}$ where $T_{T}$ is total kinetic energy, $V_{T}$ is total potential energy, $Q_{x c}$ and $Q_{\theta}$ are the generalized force applied on the generalized coordinate $x_{c}$ and $\alpha$, respectively. Both generalized forces can be defined as below:

$Q_{x c}(t)=F_{c}(t)-B_{e q} \dot{x}_{c}$

and

$Q_{\alpha}(t)=-B_{p} \dot{\alpha}(t)$

This energy is usually caused by its vertical displacement from normality (gravitational potential energy) or by a spring-related sort of displacement (elastic potential energy). In this case; there is no elastic potential energy in the system. The system potential energy is only due to gravity. The cart linear motion is horizontal and as such, never has vertical displacement. Therefore, the total potential energy is fully expressed by the pendulum's gravitational potential energy, as characterized below:

$V_{T}=M_{p} g l_{p} \cos (\alpha(t))$

The kinetic energy measures the amount of energy in a system due to its motion. Here, the total kinetic energy can be represented as follows: 
$T_{T}=T_{c}+T_{p}$

where $T_{c}$ and $T_{p}$ are the sum of the translational and rotational kinetic energies arising from both the cart and its mounted inverted pendulum, respectively. First, the translational kinetic energy of the motorized cart, $T_{c t}$, is expressed as follows:

$T_{c t}=\frac{1}{2} M \dot{x}_{c}^{2}$

Second, the rotational kinetic energy due to the cart's DC motor, $T_{c r}$, can be characterized by:

$T_{c r}=\frac{1}{2} \frac{J_{m} K_{g}{ }^{2} \dot{x}_{c}{ }^{2}}{r_{m p}{ }^{2}}$

Therefore, the cart's total kinetic energy, can be written as shown below:

$T_{c}=\frac{1}{2} M_{c} \dot{x}_{c}^{2}$

where $M_{c}=M+\left(J_{m} K_{g}^{2} / r_{m p}{ }^{2}\right)$.

The pendulum total kinetic energy, $T_{p}$ is combined of translational kinetic energy, $T_{p t}$ and rotational kinetic energy, $T_{p r}$.

$$
\begin{aligned}
T_{p} & =T_{p t}+T_{p r} \\
& =\frac{1}{2} M_{p} \dot{r}_{p}^{2}+\frac{1}{2} I_{p} \dot{\alpha}^{2}(t)
\end{aligned}
$$

where $\dot{r}_{p}{ }^{2}=\dot{x}_{p}{ }^{2}+\dot{y}_{p}{ }^{2}$. From Fig. 2, $\dot{x}_{p}$ and $\dot{y}_{p}$ can be expressed as:

$$
\dot{x}_{p}=\dot{x}_{c}-l_{p} \cos (\alpha(t)) \dot{\alpha}(t)
$$

and

$$
\dot{y}_{p}=-l_{p} \sin (\alpha(t)) \dot{\alpha}(t)
$$

Substituting (24), (25), (26), (27) into (21), gives the total kinetic energy, $T_{T}$ of the system as:

$$
\begin{aligned}
& T_{T}=\frac{1}{2}\left(M_{c}+M_{p}\right) \dot{x}_{c}{ }^{2}-M_{p} l_{p} \cos (\alpha(t), \\
& \dot{\alpha}(t) \dot{x}_{c}(t)+\frac{1}{2}\left(I_{p}+M_{p} I_{p}{ }^{2}\right) \dot{\alpha}^{2}(t)
\end{aligned}
$$

The Lagrangian can be expressed using (20) and (28):

$$
\begin{aligned}
& L=T_{T}-V_{T} \\
& =\frac{1}{2}\left(M_{c}+M_{p}\right) \dot{x}_{c}^{2}(t)-M_{p} l_{p} \cos (\alpha(t)) \dot{\alpha}(t) \dot{x} \\
& \dot{i}_{c}(t)+\frac{1}{2}\left(I_{p}+M_{p} I_{p}{ }^{2}\right) \dot{\alpha}^{2}(t)-M_{p} l_{p} \cos (\alpha(t))
\end{aligned}
$$

From equation (16) and (17), the nonlinear equation of motion can be obtained as:

$$
\begin{aligned}
& \left(M_{c}+M_{p}\right) \ddot{x}_{c}(t)+B_{e q} \dot{x}_{c}(t)-\left(M_{p} l_{p} \cos (\alpha(t))\right) \\
& \ddot{\alpha}(t)+M_{p} l_{p} \sin (\alpha(t)) \dot{\alpha}^{2}(t)=F_{c}(t)
\end{aligned}
$$

and

$-M_{p} l_{p} \cos (\alpha(t)) \ddot{x}_{c}(t)+\left(I_{p}+M_{p} l_{p}^{2}\right) \ddot{\alpha}(t)$

$+B_{p} \dot{\alpha}(t)-M_{p} g l_{p} \sin (\alpha(t))=0$

The linearized model of (30) and (31) can be obtained by considering the small variations of $\alpha$ about the equilibrium point when the pendulum is at upright position and neglecting higher order term. The linearized model can be expressed in state space representation as follows:

$\dot{\mathbf{x}}=A x+B u$

$y=C x$

where, $\quad x=\left[\begin{array}{llll}x_{c} & \alpha & \dot{x}_{c} & \dot{\alpha}\end{array}\right]^{T} \quad, \quad u=F_{c} \quad$ and $y=\left[\begin{array}{ll}x_{c} & \alpha\end{array}\right]^{\mathrm{T}}$ and matrix $A, B$ and $C$ are given as:

$A=\left[\begin{array}{cccc}0 & 0 & 1 & 0 \\ 0 & 0 & 0 & 1 \\ 0 & \frac{\left(M_{p} l_{p}\right)^{2} g}{\mathrm{Z}} & -\frac{B_{e q}\left(M_{p} l_{p}{ }^{2}+I\right)}{\mathrm{Z}} & -\frac{M_{p} l_{p} B_{p}}{\mathrm{Z}} \\ 0 & \frac{\left(M_{p}+M_{c}\right) M_{p} g l_{p}}{\mathrm{Z}} & -\frac{M_{p} l_{p} B_{e q}}{\mathrm{Z}} & -\frac{\left(M_{p}+M_{c}\right) B_{p}}{Z}\end{array}\right]$

$B=\left[\begin{array}{c}0 \\ 0 \\ \frac{\left(I+M_{p} l_{p}{ }^{2}\right)}{Z} \\ \frac{M_{p} l_{p}}{Z}\end{array}\right] \quad C=\left[\begin{array}{llll}1 & 0 & 0 & 0 \\ 0 & 1 & 0 & 0\end{array}\right]$

where $Z=\left(M_{c}+M_{p}\right) I_{p}+M_{c} M_{p} l_{p}{ }^{2}$

SESIP control strategy model: The control strategy to implement the SESIP consists of two main control loops and a decision-making logic component to switch between the two. One control loop is a PV controller on the cart position in order to swing up the single pendulum from the suspended to the inverted posture. The other control loop is active when the pendulum is around the upright position and consists of maintaining the inverted pendulum vertical. It uses a TS fuzzy controller with ANFIS architecture.

Swing-up controller design: To control the cart, a control strategy based on the proportional-velocity (PV) control scheme is used in order to to make the closedloop system to satisfy the following performance requirements:

* The percent overshoot (PO) should be less than $10 \%$ i.e. $\mathrm{PO}<10 \%$

* The time to first peak should be $150 \mathrm{~ms}$ i.e. $t_{p}=$ $0.15 s$.

The PV control law can be expressed as follows:

$V_{m}(t)=K_{p}\left(x_{d}(t)-x_{c}(t)\right)-K_{v} \dot{x}_{c}(t)$

where $x_{d}$ is the reference signal i.e. the desired position to track. Then, the closed-loop transfer function can be expressed as: 


$$
\frac{x_{c}(s)}{x_{d}(s)}=\frac{K_{p} G(s)}{1+K_{p} G(s)+G(s) K_{v} s}
$$

Replacing $G(s)$ in equation (15) into (34) and using model parameter values in Table 1 leads to the following:

$$
\frac{x_{c}(s)}{x_{d}(s)}=\frac{2.46 K_{p}}{s^{2}+\left(2.46 K_{v}+17.13\right) s+2.46 K_{p}}
$$

According to performance requirements, $\mathrm{PO}=10 \%$ and $t_{p}=0.15 \mathrm{~s}$, a natural frequency, $\omega_{n}=26.0 \mathrm{rad} / \mathrm{s}$ and damping ratio, $\zeta=0.59$ can be obtained. The characteristic equation of the closed-loop transfer function expressed in its standard form is as follows:

$$
s^{2}+2 \zeta \omega_{n} s+\omega_{n}^{2}
$$

Comparing (35) and (36) lead to the following PV controller gains:

$K_{p}=274.62 \mathrm{~V} / \mathrm{m}$ and $K_{v}=5.53 \mathrm{Vs} / \mathrm{m}$.

Stabilizing controller design: Based on (32), the Takagi-Sugeno (TS) fuzzy controller develop here has four inputs i.e. $x_{c}, \alpha, \dot{x}_{c}$ and $\dot{\alpha}$ and a single output i.e. $V_{m}$. These four input variables considerably affect the control action, $V_{m}$ which is applied to the cart motor. The control problem here is to design a controller that maps the state vector $x$ into an appropriate control action, $V_{m}$, such that the pendulum is kept balanced while the cart is tracking back to its origin of track. In addition, the choice of membership functions (MFs) and rule base of the TS fuzzy controller will affect the performance of the system. The TS fuzzy controller with ANFIS architecture can formalize a systematic approach to generate the fuzzy rule and MFs.

Training data set: The training data set that contains the desired input/output data pairs of the actual plant is obtained through the real-time experiments. Figure 2 and 3 show block diagram of real-time implementation for collecting input-output data pairs of the inverted pendulum which is in the following format:

$$
[\underbrace{x_{c}, \quad \alpha, \quad \dot{x}_{c}, \quad \dot{\alpha}}_{\text {input } \text { variables }} \underbrace{V_{m}}_{\text {output }} \text { variable }]
$$

Also from the experiment, we manage to get 100 sets of checking data of similar format as above.

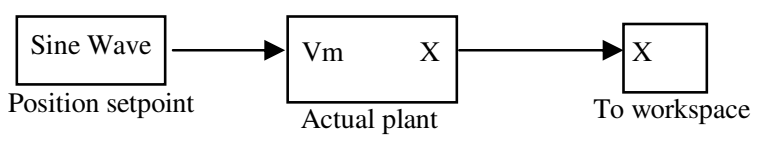

Fig. 2: Block diagram of real-time implementation

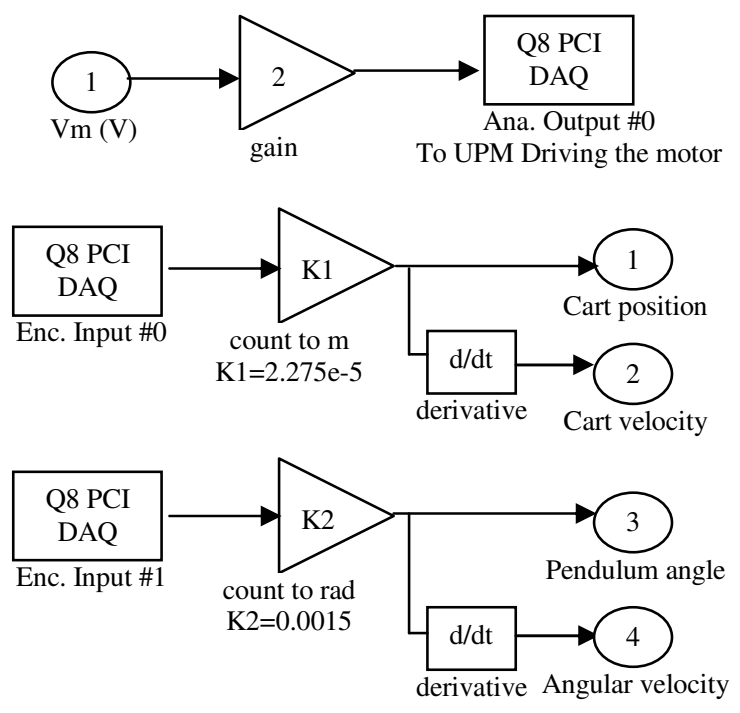

Fig. 3: Actual plant subsystem
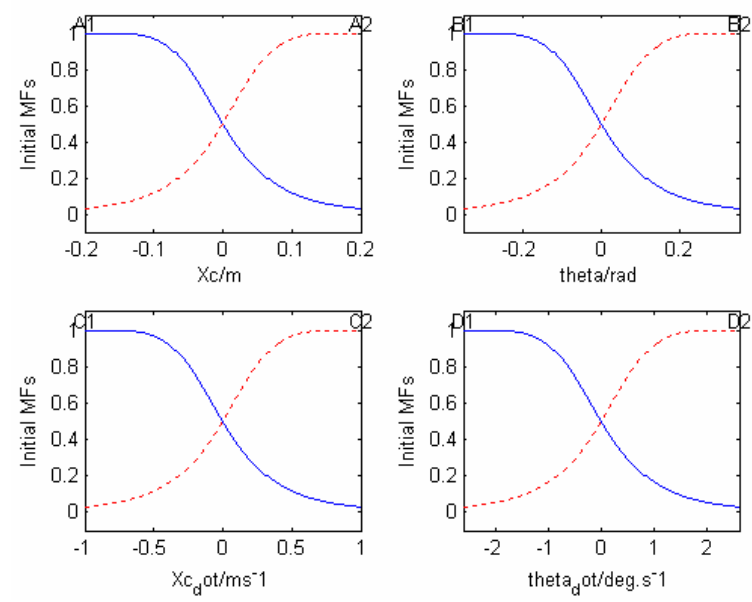

Fig. 4: Initial membership functions for fuzzy controller

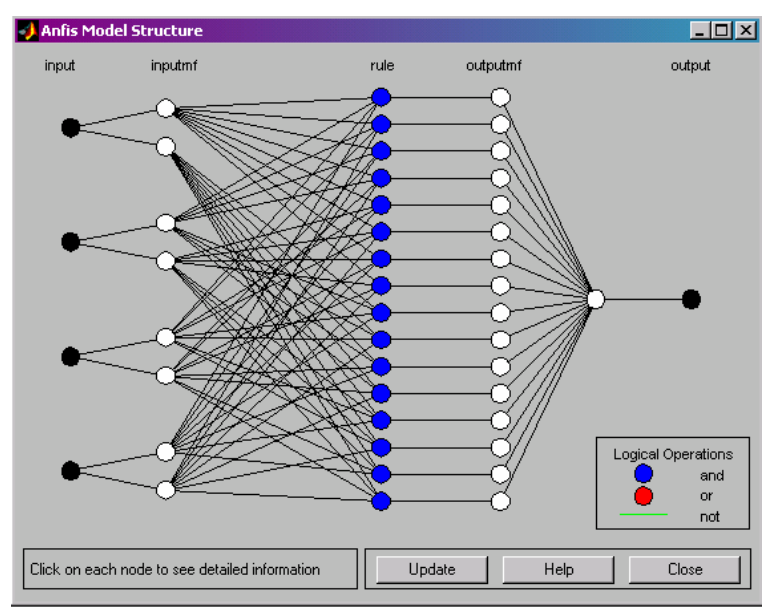

Fig. 5: ANFIS model structure

Initial TS fuzzy inference system: The initial linear relationship between input and output are described in 

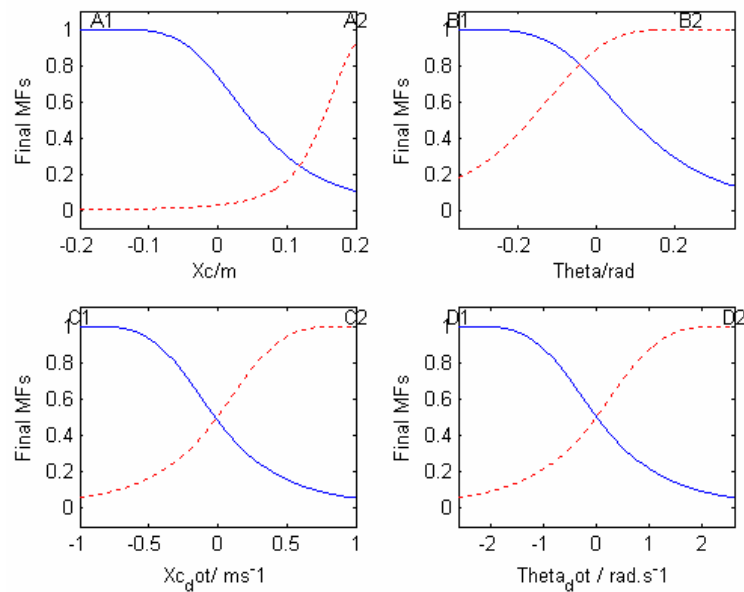

Fig. 6: Final membership functions for ANFIS controller
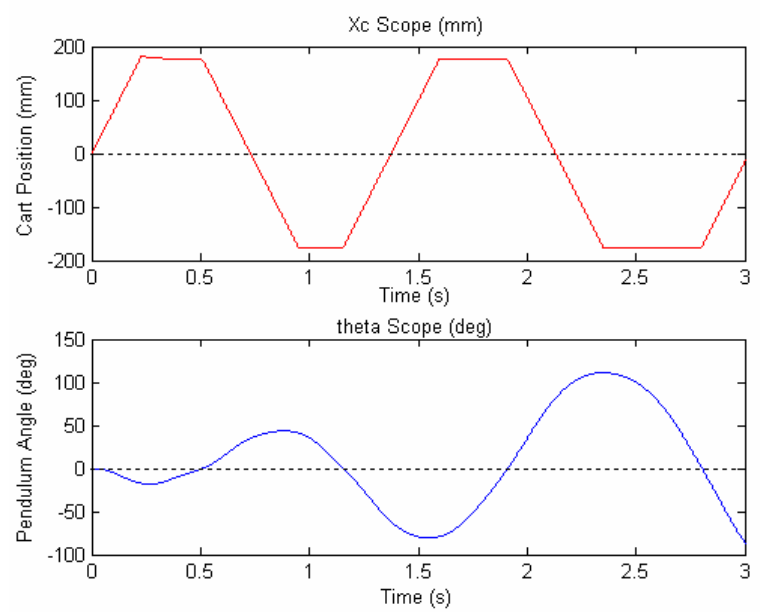

Fig. 7: Simulation results for swing-up control

16 fuzzy-if-then rules which are represented in the format as:

Rule $i$ : IF $\left(x_{c}\right.$, is $\left.\mathrm{A}_{j}\right)$ and $\left(\alpha\right.$ is $\left.\mathrm{B}_{j}\right)$ and $\left(\dot{x}_{c}\right.$ is $\left.\mathrm{C}_{j}\right)$ and

( $\dot{\alpha}$ is $\mathrm{D}_{j}$ ),

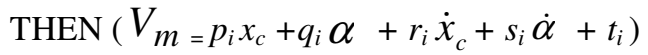

where $i=1,2, \ldots, 16 ; j=1,2$.

The input MFs for initial fuzzy inference system (FIS) in (37) is shown in Fig. 4.

Trained TS fuzzy inference system: Figure 5 shows ANFIS model structure using ANFIS GUI editor from MathWork Inc. During the training process, the hybrid method with the combination of backpropagation and least square method is implemented to estimate MFs parameters.

Finally, a trained FIS structure is created from the initial FIS using ANFIS GUI editor and training and checking data sets. The MFs of trained FIS are shown in Fig. 6. The output parameters and rule base of trained data cannot be shown here due to limited space.

\section{Simulation}

Swing-up control simulation: From Fig. 7, the cart is moving alternately between $180 \mathrm{~mm}$ and $-180 \mathrm{~mm}$ to increase the energy of the system as quickly as possible by moving the cart to its maximum allowed distance of track (i.e. $0.2 \mathrm{~m}$ ). In this swing-up mode, PV controller destabilizes the pendulum when initially at rest and hanging down. The amplitude of the pendulum angle become larger until it is close to the upright position, so that the stabilizing controller will catch the pendulum and balance it later. As can be seen in the simulation results, the PV controller takes $2.5 s$ for 4 swings. Thus, the PV controller could be effectively employed in SESIP control system as a swing-up controller to get maximum swing in the shortest possible time.

Stabilizing control simulation: Figures 8 and 9 show control results of the inverted pendulum system by Simulink simulation.

In Fig. 9, the initial angle of the pendulum is 10 degree and the other initial values are all zeros. Since the initial value of the pendulum is positive to the left, the cart is first driven from the original position to the left side (which is defined as negative) such that the pendulum rotates clockwise towards upright position. After the pendulum reaches the upright position, it still rotates to the negative (to the right) direction because of its inertia energy of movement. Then the negative driven force moves the cart back towards the origin, causing the pendulum to stand up eventually. It takes $2.75 s$ to be stabilized. In Fig. 10, the cart is applied an input of square-wave signal with $20 \mathrm{~mm}$ of amplitude. The signal frequency is set to $0.1 \mathrm{~Hz}$. The pendulum system is managed to be stabilized in upright position, even though the disturbance is applied to the cart position alternately. In this case, the robustness of the SESIP system is successfully realized by the proposed ANFIS controller.

\section{RESULTS}

Real-time experiment configuration consists of computer with MATLAB, Simulink and Quanser Toolbox used as a controller, Q8 data acquisition board and Quanser IP02 Linear Motion Servo Module. Some hardware limitations should be concerned in the cartpendulum system. The Digital-to-Analog voltage for data acquisition board is limited between $-10 \mathrm{~V}$ and 10 V. The safety watchdog is turned on where the allowable cart displacement is $0.35 \mathrm{~m}$ from the centre of the track. When the pendulum or cart touches the limit switch, the control process is aborted. Figures 11 to 13 show the SESIP control system experimental results.

It is important to note that the PV controller takes approximately 3.5 seconds to reach the upright position. The point at which the ANFIS controller catches the pendulum in the upright position is clearly shown in the above plots. 


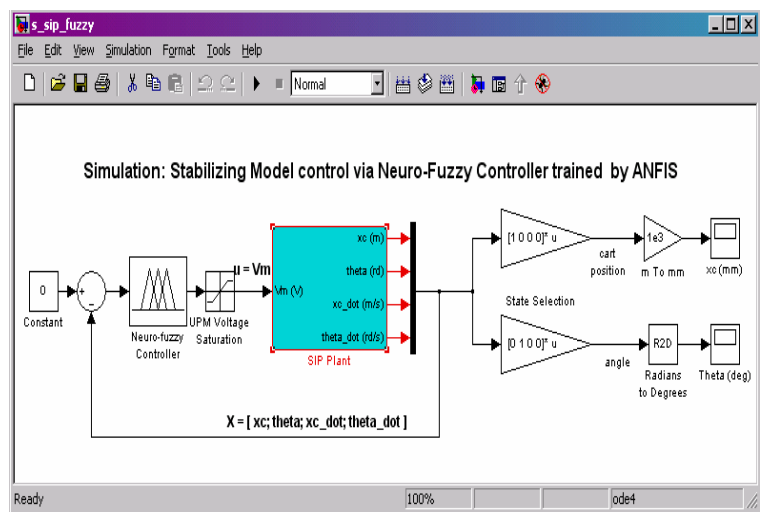

Fig. 8: Simulink diagram of balance control
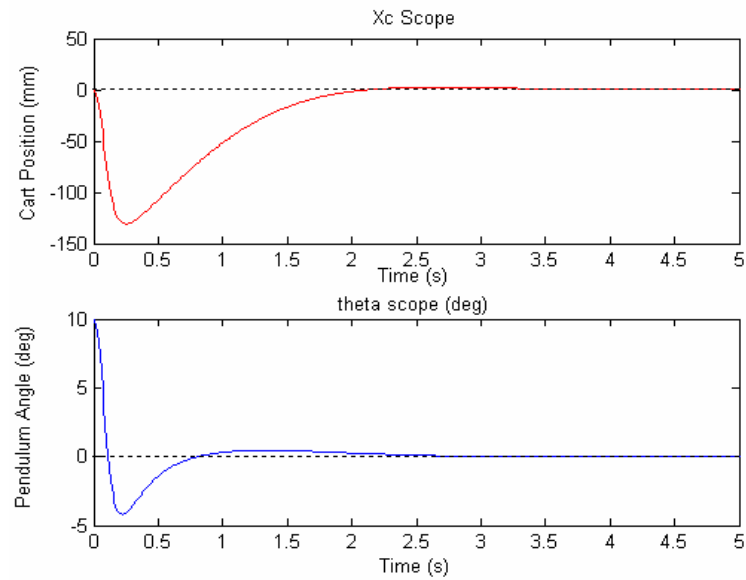

Fig. 9: Simulation results for stabilization control
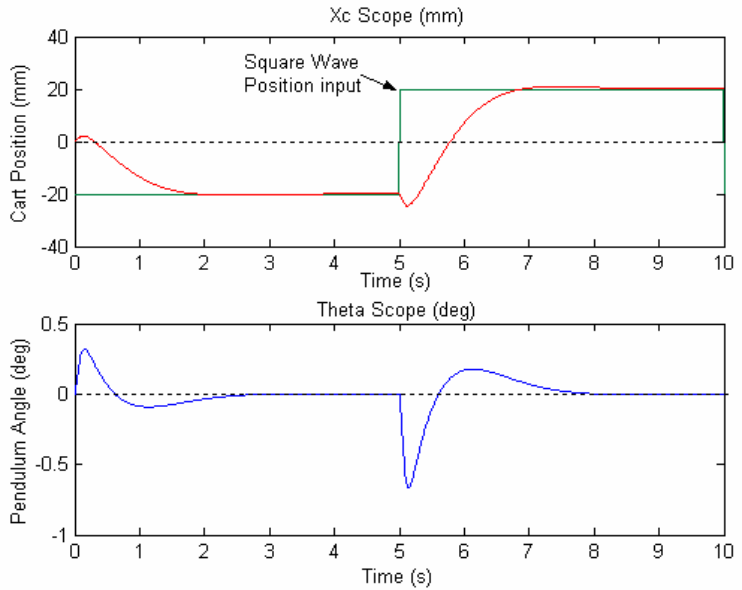

Fig. 10: Simulation results for stabilization control when system is applied square-wave position input

Figure 11 shows that the control output, $V_{m}$ for control of SESIP system is not constant all the way. Initially maximum $V_{m}$ that is $10 \mathrm{~V}$ (in our experiment, the voltage saturation is limited to be $10 \mathrm{~V}$ ) supplied to the cart motor in its attempt to start the pendulum from rest. Then the voltage decreases a bit when the pendulum is falling naturally by the gravitational

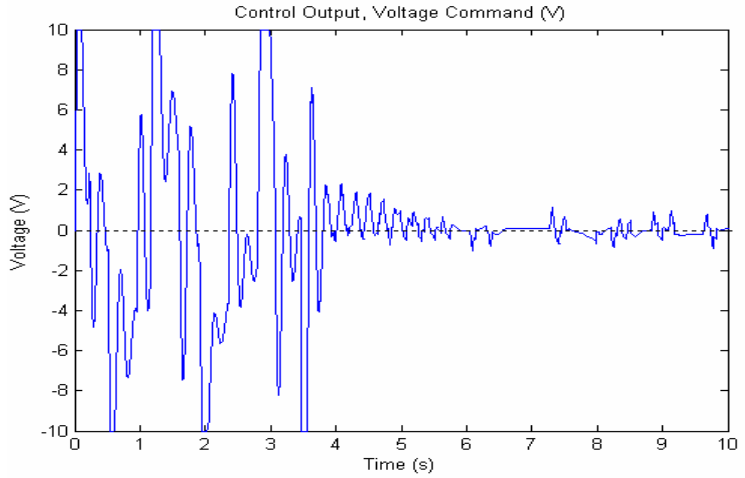

Fig. 11: Voltage output for cart motor

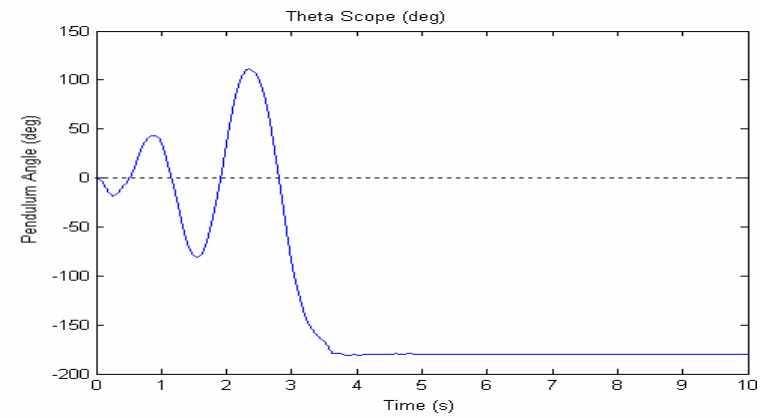

Fig. 12: Experimental result of pendulum angle, $\theta$

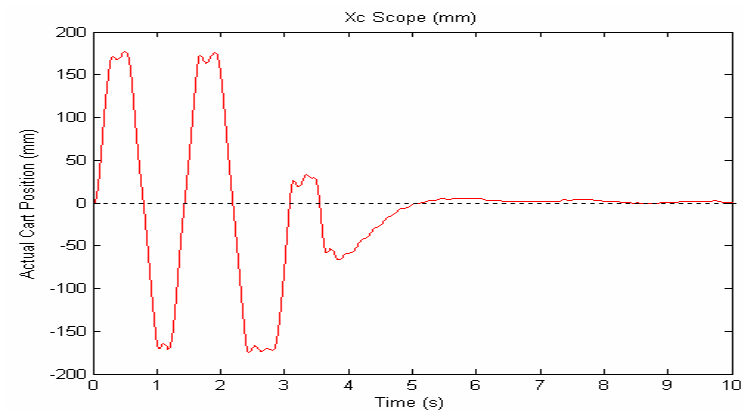

Fig. 13: Experimental result of cart position, $x_{c}$

potential energy. To regain energy to swing up the pendulum, the maximum voltage is supplied to the cart again. This phenomenon continues until the pendulum reaches within 15 degree from the upright position. The trend of voltage command explained above is fed by PV controller of swing-up mode. Once the stabilizing controller is activated and catches the pendulum, the output voltage starts to decrease greatly in magnitude because less energy is needed to control the small deviation of pendulum in balance mode. In this case, the voltage supplied is in the range of $\pm 1 \mathrm{~V}$ which is controlled by ANFIS controller. The corresponding plot of the pendulum angle is shown in Fig. 12. Each swing increases the pendulum angle slightly until the pendulum is closed to the upright position, which is defined to be 180 degree from its initial hanging down position. At that stage, the pendulum angle remains fairly constant. Note that the controller takes about 4 swings to bring the pendulum close enough to upright 
position within $3.5 \mathrm{~s}$. After that, it remains stabilized throughout the experimental period for no matter how long it is. It is indicated that the switch is triggered effectively so that the ANFIS controller could catch the pendulum and balance it completely. Figure 13 depicts the cart linear position for the entire control process. Initially, the cart linear displacement alternates between $180 \mathrm{~mm}$ and $-180 \mathrm{~mm}$.

As can be seen, a few cart large movements are done, until about $3 s$, the pendulum amplitude of oscillation becomes large enough to be beyond 150 degree from the downward position. Then, it takes the smaller cart position set point amplitude about another $0.5 s$ to slowly bring the pendulum close to the upright position. Once the pendulum is almost balanced, the cart will return to its origin of track within $1.5 \mathrm{~s}$, which is at $5 s$ of the experimental time. Slight errors appear at the maximum displacement of the cart because this is the time when the cart abruptly changes its direction.

Consequently, as can be seen from Fig. 12 and 14, the control force optimized by PV controller is able to move the cart back and forth with a minimum number of times and finally bring the pendulum up as quickly (and smoothly) as possible. The stabilization control of the pendulum is also successfully realized in real time by the proposed ANFIS controller.

\section{CONCLUSION}

The objective of this project was to design a stabilizing controller for SESIP problem and this has been successfully achieved. The simulation and experimental results show that the hybrid controllers take about $3.5 s$ and 4 swings to bring the pendulum close to upright position while the stabilizing controller with only 16 fuzzy rules is able to balance the pendulum for the rest of the experiment period. It is worth to note that the cart is able to return to the origin of the rail after the pendulum is stabilized. This controller is proved to be effective and feasible in both of the angular control of pendulum at upright position and position control of cart to its origin of rail.

\section{REFERENCES}

1. Mikulcic, A. and J. Chen, 1996. Experiments on using fuzzy clustering for fuzzy control system design. Proc. FUZZ-IEEE'96, 3: 2168-74.

2. Brock, S., 2003. Practical approach to fuzzy control of inverter pendulum. IEEE Intl. Conf. Industrial Technology'03, 1: 31-35.

3. Kandadai, R.M. and J.M. Tien, 1996. On a fuzzyneural hierarchical controller with a self-generating knowledge base. Proc. IEEE Intl. Conf. on Systems, Man and Cybernetics, 4: 2625-2630.

4. Kawaji, S. and T. Maeda, 1991. Fuzzy servo control system for an inverted pendulum. Proc. Internet Fuzzy Engineering Symp., 2: 812-823.

5. Horikawa, S.I., T. Furuhashi and Y. Uchikawa, 1992. On fuzzy modeling using neural networks with the back propagation algorithm. IEEE Trans. Neural Networks, 3: 801-806.

6. Lin, C.T. and C.S.G. Lee, 1992. Neural network based fuzzy logic control and decision systems. IEEE Trans. Computers, 40: 1320-1336.

7. Patricar, A. and J. Provence, 1990. A selforganizing controller for dynamic processes using neural networks. Intl. Joint Conf. Neural Networks, 3: 359-364.

8. Takagi, H. and I. Hayashi, 1991. NN-driven fuzzy reasoning. Intl. J. Approximate Reasoning, 5: 191212.

9. Kyung, K.H. and B.H. Lee, 1993. Fuzzy rule base derivation using neural network based fuzzy logic controller by self-learning. Proc. IECON'93, 1: 435-440.

10. Sakai, S. and T. Takahama, 1997. Learning fuzzy control rules for inverted pendulum by simplex method. Proc. of the13th Fuzzy System Symposium, pp: 61-64.

11. Jang, J.S., 1993. ANFIS: Adaptive-network-based fuzzy inference system. IEEE Trans. Systems, Man and Cybernetics, 23: 665-685. 